\title{
Scalable High-Speed Hybrid Complementary Integrated Circuits based on Solution-Processed Organic and Amorphous Metal Oxide Semiconductors
}

\section{Shun Watanabe ( $\nabla$ swatanabe@edu.k.u-tokyo.ac.jp )}

University of Tokyo https://orcid.org/0000-0001-7377-6043

\section{Xiaozhu Wei}

University of Tokyo

\section{Shohei Kumagai}

The University of Tokyo

\section{Tatsuyuki Makita}

University of Tokyo

Kotaro Tsuzuku

University of Tokyo

Akifumi Yamamura

University of Tokyo

\section{Mari Sasaki}

University of Tokyo

Jun Takeya

University of Tokyo https://orcid.org/0000-0002-7003-1350

\section{Article}

Keywords: Scalable High-Speed Hybrid Complementary Integrated Circuits, Organic and Amorphous Metal Oxide Semiconductors, thin-film transistors (TFTs)

Posted Date: September 13th, 2021

DOl: https://doi.org/10.21203/rs.3.rs-883006/v1

License: (9) (1) This work is licensed under a Creative Commons Attribution 4.0 International License. Read Full License 


\title{
Scalable High-Speed Hybrid Complementary Integrated Circuits based on Solution- Processed Organic and Amorphous Metal Oxide Semiconductors
}

Xiaozhu Wei, Shohei Kumagai, * Tatsuyuki Makita, Kotaro Tsuzuku, Akifumi Yamamura, Mari Sasaki, Shun Watanabe*, and Jun Takeya

Material Innovation Research Center and Department of Advanced Materials Science, Graduate School of Frontier Sciences, University of Tokyo, 5-1-5 Kashiwanoha, Kashiwa, Chiba 277-8561, Japan

*Corresponding author

Telephone: +81-4-7136-3788; e-mail: swatanabe@edu.k.u-tokyo.ac.jp

\begin{abstract}
Solution-processed single-crystal organic semiconductors (OSCs) and amorphous metal oxide semiconductors (MOSs) are promising for high-mobility, p- and n-channel thin-film transistors (TFTs), respectively. Organic-inorganic hybrid complementary circuits hence have great potential to satisfy practical requirements; however, some chemical incompatibilities between OSCs and MOSs, such as heat and chemical resistance, conventionally make it difficult to rationally integrate TFTs based on solution-processed OSC and MOS into the same substrates. In this work, we achieved a rational integration method based on the solution-processed semiconductors by carefully managing the device configuration and the deposition and patterning techniques from materials point of view. The balanced high performances as well as the uniform fabrication of the TFTs led to densely integrated five-stage ring oscillators with the stage propagation delay of $1.3 \mu \mathrm{s}$, which is the fastest operation among ever reported complementary ring oscillators based on solutionprocessed semiconductors.
\end{abstract}




\section{Introduction}

Solution technique is of vital interest to both industry and academia for manufacturing electronic devices. To meet the requirement from the future Internet of Things (IoT) society, solution techniques suitable for thin-film transistors (TFTs) on plastic substrates get more and more attention, where the solution-processed deposition of semiconductors is the primary subject. From engineering point of view, complementary integrated circuits (ICs), which are constructed from the pairs of p- and n-channel TFTs, with high-mobility and performancebalanced p- and n-channel TFTs are preferred for actual devices such as radio-frequency identification tags and sensors because they can enable high operation speeds, low power dissipation, wide noise margin, and an easy and compact circuit design. For this aim, organic semiconductors (OSCs) are the most promising for the TFT applications owing to their solubility, low fabrication temperature and high compatibility with flexible substrates ${ }^{1}$. Thus far, OSC materials and solution techniques have been developed to facilitate the implementation of organic TFTs, particularly with single-aligned crystals $^{2-8}$, which could be beneficial for high electrical properties. Despite the advantages of complementary ICs, most of solution-processable and high-mobility OSCs predominantly behave as hole-transporting materials, thus providing p-channel TFTs. In other words, (so-called n-type) OSCs suitable for n-channel TFTs are still lagging behind p-type counterparts due to their anisotropic crystal packings and less effective electronic couplings due to the essential introduction of strong electron-withdrawing groups ${ }^{9}$. Meanwhile, thin films of amorphous metal oxide semiconductors (MOSs) can be also prepared by solution processes, such as the sol-gel method, and have been developed for $n$-channel TFTs ${ }^{10-15}$ because of their high electron mobility, excellent uniformity, and good ambient stability. By contrast to OSCs, MOSs are hardly useful for p-channel TFTs owing to the unfavourable hole formation as well as holetransporting path due to the presence of highly occupied localised oxygen $2 p$ orbitals ${ }^{16}$. Most 
of the reported ICs based on either only solution-processed OSCs or MOSs are hence designed by unipolar or pseudo-complementary operations ${ }^{17-21}$, or complementary circuits with an unsatisfactory performances ${ }^{22-27}$.

Therefore, hybridisation is one way to overcome the limitations faced by a single material and this approach has been used to build complementary circuits ${ }^{28-30}$. However, organic-inorganic hybrid complementary ICs based on solution-processed OSC and MOS, employed for p- and n-channel TFTs, respectively, have been rarely demonstrated despite their promises, although single complementary inverters have often been reported ${ }^{29-32}$. This is probably due to the different and complex chemical characteristics of the semiconducting materials being integrated into the same circuit. For instance, OSCs are self-assembled in the solid states via weak van der Waals forces, whereas MOSs are covalently bound, which leads to incompatible processing parameters, such as temperature, heat and chemical resistance, and adaptability to lithographic processes. Due to the curing conditions, MOSs are prepared in advance of OSC deposition, which often deteriorates the MOSs maybe due to organic solvent vapor and heat during the OSC deposition. In addition, fine patterning is mandatory in both semiconductors and electrodes to avoid crosstalk and realise high-speed operations, and the requirement of a high degree of integration for practical application affords further tasks; therefore, the complementary ICs based on solution-processed, high-performance OSC and MOS are challenging. Although a few solution-processed hybrid complementary ICs have been demonstrated with photolithographic processes, their TFT performances, such as mobility and on-off switching ratio, were compromised because of the low mobility of macromolecular OSCs and the chemical degradation of MOSs ${ }^{33,34}$. In other words, there were no rational and feasible methodologies to integrate solution-processed OSCs and MOSs into complementary ICs despite the very simple concept of hybridisation. 
In this study, we demonstrate the hybrid complementary ICs composed of solution-processed high-performance semiconductors: single crystals of a small-molecule OSC, 3,11dinonyldinaphto[2,3-d:2',3'-d']benzo[1,2-b:4,5-b']dithiophene (C9-DNBDT-NW), and amorphous indium zinc oxide (IZO) for p- and n-channel TFTs, respectively. They were selected because of their high carrier mobility, uniformity, and potential scalability ${ }^{7,35,36}$. A technology was developed to realize high-speed operations with careful management of an elimination of the chemical compatibility issues during the patterning process and an exclusion of the damage on MOS-based TFTs from further integration. In the following sections, we will describe our achievements based on the hybrid complementary inverters, which exhibit desirable switching properties and excellent long-term stability on a plastic substrate. Demonstration of five-stage complementary ring oscillators revealed the uniformity of both single-crystal $\mathrm{C}_{9}$-DNBDT-NW and amorphous IZO, and the short propagation delay of $1.3 \mu$ s per stage with an operation voltage of $10 \mathrm{~V}$ was achieved, indicating that the present facile method combining the advantages of solution-processed OSC and MOS can meet future IoT demands.

\section{Results and discussion}

Integrated process. The hybrid complementary inverter on a polyimide (PI) substrate with $\mathrm{C}_{9}$-DNBDT-NW single crystals and amorphous IZO as the p- and n-channel material, respectively, is schematically illustrated in Figure 1. Both the p- and n-channel TFTs have bottom-gate top-contact structures. As illustrated in Figure S1, for the IZO-based n-channel TFT, gate electrodes were fabricated by photolithography and a lift-off process. Meanwhile, the $\mathrm{AlO}_{\mathrm{x}}$ gate dielectric layer was formed by atomic layer deposition (ALD). The IZO layer was deposited by spin coating and patterned via photolithography and wet-etching. To reduce the number of photolithography steps, source/drain (S/D) electrodes of $n$-channel TFTs and gate electrodes of p-channel TFTs were fabricated simultaneously. Then, 
polymethylmethacrylate (PMMA) and parylene were sequentially formed as a bilayer gate dielectric for p-channel TFTs and as a passivation layer for n-channel TFTs to protect the back-channel of IZO-based TFTs against potential damage during subsequent integration processes, where PMMA could protect IZO from undesired chemical reaction with the monomeric diradical intermediate of parylene ${ }^{37}$. C9-DNBDT-NW was formed using a scalable printing technique, namely, continuous edge casting method ${ }^{7,38}$, and then transferred to the top of the PMMA/parylene dielectric layer ${ }^{39}$.



Figure 1 Schematic structure of a hybrid complementary inverter with IZO as the n-channel material and $\mathrm{C}_{9}$-DNBDT-NW single crystal as the p-channel material

A cross-polarised optical micrograph of $\mathrm{C}_{9}$-DNBDT-NW single crystals on the as-fabricated IZO-based TFTs before patterning revealed a clear crystal domain (Figure S2). Scanning electron microscopy (SEM) images of the cross-section of the $\mathrm{C}_{9}$-DNBDT-NW channel produced by focused ion beam (FIB) machining revealed burr-free gate electrodes of the pchannel TFT (Figure S3), which was a key to the successful fabrication of the $\mathrm{C}_{9}$-DNBDTNW single crystal layer. OSC patterns and Au S/D electrodes were formed via a two-step patterning process, in which photosensitive dielectric materials (PDMs, a dry-film photoresist)/PMMA double sacrificial layers were employed for a damage-free patterning of 
OSC-based TFTs. As shown in Figure S4, PDM (the top layer) enables the formation of the OSC and S/D patterns while PMMA facilitated the stripping of the resist with acetonitrile, thus causing little damage to the OSC. The PDM was laminated on the substrate and patterned by photolithography ${ }^{40,41}$, while the PMMA layer was deposited via spin coating and etched with $\mathrm{O}_{2}$ plasma. Furthermore, the PDM contributed to the damage-free fabrication of OSC-based TFTs as the solvent content in the dry film was less than $2 \mathrm{wt} . \%$ and the PDM patterning process is based on the polymerization of double bonds rather than the generation of photoacids. Therefore, the potential damage induced by solvents or acids in conventional photolithography could be eliminated effectively. The contact resistance $\left(R_{\mathrm{c}}\right)$ of $\mathrm{C}_{9}$-DNBDTNW-based TFTs fabricated by this technology was studied using the transfer-line method, as described in the Supporting Information (Section S1). The intrinsic mobility $\left(\mu_{\text {int }}\right)$ of $C_{9-}$ DNBDT-NW TFTs was $\sim 10 \mathrm{~cm}^{2} \mathrm{~V}^{-1} \mathrm{~s}^{-1}$ and the normalised contact resistance $\left(R_{\mathrm{c}} W\right)$ was $\sim 230 \Omega \mathrm{cm}$ (Figure S5), which is a low value for OSCs without extra doping ${ }^{6}$; this implies a high effectiveness of carrier injection in the fabricated $\mathrm{C}_{9}$-DNBDT-NW-based TFTs.

Electrical performance of the hybrid inverters. A micrograph of a complementary inverter is shown in the inset of Figure 2(a). The output curves revealed that both the n- and p-channel TFTs exhibited a typical output with good pinch-off behaviour and negligible hysteresis (Figure 2(a)). The transfer characteristics of each TFT in the linear and saturation regions are illustrated in Figure 2(b)-(e). In the case of $\mathrm{C}_{9}$-DNBDT-NW-based TFTs, transfer curves in the linear (Figure 2(b)) and saturation (Figure 2(d)) regions indicated the linear and saturation mobilities $\left(\mu_{\text {lin }}\right.$ and $\mu_{\text {sat }}$, respectively), on-off current ratio $\left(I_{\mathrm{on}} / I_{\mathrm{off}}\right)$, off current $\left(I_{\mathrm{off}}\right)$, and turn-on voltage $\left(V_{\mathrm{on}}\right)$ of 5.8 and $5.1 \mathrm{~cm}^{2} \mathrm{~V}^{-1} \mathrm{~s}^{-1}, 10^{8}, 10^{-12} \mathrm{~A}$, and $\sim 2 \mathrm{~V}$, respectively; the corresponding values in the IZO TFT were 2.9 and $4.0 \mathrm{~cm}^{2} \mathrm{~V}^{-1} \mathrm{~s}^{-1}, 10^{8}, 10^{-12} \mathrm{~A}$ and $\sim 0 \mathrm{~V}$, respectively (Figure 2(c) and (e)). 
(a)


Figure 2 Electrical performance of $\mathrm{C}_{9}$-DNBDT-NW- and IZO-based TFTs. (a) Output performance. The inset shows a micrograph of a complementary inverter with channel width $(W) /$ length $(L)=200 \mu \mathrm{m} / 9 \mu \mathrm{m}$ for the C $_{9}$-DNBDT-NW p-channel and $200 \mu \mathrm{m} / 13 \mu \mathrm{m}$ for the IZO n-channel. (b)-(e) Transfer characteristics in the linear region and the saturation region. $V_{\mathrm{D}}$ : Drain voltage.

Due to contact resistance, the effective mobility $\left(\mu_{\text {eff }}\right)$ is lower than $\mu_{\text {int }}$, especially in shortchannel devices, as shown in Equation (1),

$$
\mu_{\mathrm{eff}}=\mu_{\mathrm{int}} \frac{1}{1+\frac{R_{\mathrm{C}} W}{L} \mu_{\mathrm{int}} C_{\mathrm{i}}\left(V_{\mathrm{G}}-V_{\mathrm{th}}\right)}
$$


where $C_{\mathrm{i}}$ is the gate capacitance per unit area, $V_{\mathrm{G}}$ represents gate voltage, and $V_{\text {th }}$ is the threshold voltage. The $\mu_{\text {eff }}$ of the $\mathrm{C}_{9}$-DNBDT-NW-based TFT was estimated to be $\sim 5.9 \mathrm{~cm}^{2}$ $\mathrm{V}^{-1} \mathrm{~s}^{-1}$, which is similar to the experimental value. In the case of the IZO-based TFT, $R_{\mathrm{C}}$ was estimated to be $29 \Omega \mathrm{cm}$ and the effective resistance measured after the integration process is


NW- and IZO-based TFTs can be attributed to the high performance of the semiconductor materials and the well-designed integration processes. Patterned gate electrodes and short channels lead to degraded performance ${ }^{42}$, especially in TFTs with bottom-gate top-contact structures. This may be because 1) as the channel length decreases, contact resistance starts to dominate, 2) the S/D patterning process may damage the semiconductor layer, and 3) the uneven surface caused by patterned gate electrodes may affect the uniformity of the active layer in the solution-processed semiconducting thin film. In this study, these problems were solved by using damage-free processes for both $\mathrm{p}$ - and n-channel TFTs, controlling the thickness and edge conditions of the gate electrode, and excluding the damage from further $\mathrm{p}$ channel TFT integration process on IZO-based TFTs, which was processed with a bilayer PMMA/parylene passivation structure.

The hybrid complementary inverter exhibited the full rail-to-rail output swing and negligible hysteresis (Figure 3(a),(b)). The corresponding voltage gain and static current $\left(I_{\text {supply }}\right)$ values are shown in Figure 3(c) and (d), respectively. At a $V_{\mathrm{DD}}$ of $7 \mathrm{~V}$, a nearly symmetrical voltage transfer curve (VTC) with a midpoint voltage $\left(V_{\mathrm{M}}\right)$ of $3.42 \mathrm{~V}$ and maximum voltage gain of $38 \mathrm{~V} / \mathrm{V}$ was observed. $I_{\text {supply }}$ was $1 \times 10^{-9} \mathrm{~A}$ at $V_{\text {in }}=0 \mathrm{~V}$ while $I_{\text {supply }}$ was on the order of $10^{-7}$ A at high $V_{\text {in }}($ Figure 3(d)), where the static power consumption was still less than $0.76 \mu \mathrm{W}$ at $V_{\mathrm{DD}}=7 \mathrm{~V}$, for example. The higher static $I_{\text {supply }}$ at high $V_{\text {in }}$ was due to higher $I_{\mathrm{D}}$ of the pchannel TFT at $V_{\mathrm{G}}=0 \mathrm{~V}$ or $V_{\text {in }}=7 \mathrm{~V}$ than that of the n-channel TFT at $V_{\mathrm{G}}=0 \mathrm{~V}$ or $V_{\text {in }}=0 \mathrm{~V}$ by 5 orders of magnitude $\left(3 \times 10^{-7}\right.$ and $1 \times 10^{-12} \mathrm{~A}$, respectively) (Figure S6). Our future 
work will aim to control $V_{\text {on }}$ for p-channel TFTs to further lower the static power consumption of complementary inverters. Figure 3(e) shows the VTC obtained with a $V_{\mathrm{DD}}$ of $7 \mathrm{~V}$; the noise margins high $\left(\mathrm{NM}_{\mathrm{H}}\right)$ and noise margin low $\left(\mathrm{NM}_{\mathrm{L}}\right)$ are indicated by green rectangles $\left(\mathrm{NM}_{\mathrm{H}}=2.7 \mathrm{~V}\right.$ and $\left.\mathrm{NM}_{\mathrm{L}}=1.9 \mathrm{~V}\right)$

It is particularly important to note that the hybrid complementary inverter exhibited a decent performance even after exposure to the ambient atmosphere (air) for 5 months (Figure 3(f)), whereas the off current slightly increased in both TFTs (Figure S7). This may be attributed to a self-healing effect in which the number of carrier traps decreased slightly over time ${ }^{6,43}$. Besides, $V_{\text {on }}$ of the IZO TFT slightly shifted, which would be due to the diffusion of environmental molecules such as oxygen and water because the current passivation layer, PMMA/parylene, could not isolate the device completely ${ }^{37}$. The passivation technique is being studied to further stabilise these devices. 
(a)



(c)



(e)



(b)

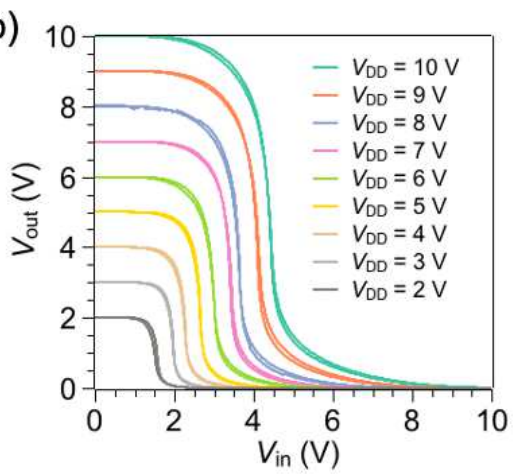

(d)



(f)



Figure 3 Electrical performance of the hybrid complementary inverter. (a) Schematic diagram. (b) Voltage transfer curves (VTCs), (c) voltage gains, and (d) static current at $V_{\mathrm{DD}}=$ 2-10 V. (e) VTCs at $V_{\mathrm{DD}}=7 \mathrm{~V}$ with noise margins represented by the green rectangles. $V_{\mathrm{M}}=$ $3.42 \mathrm{~V}, \mathrm{NM}_{\mathrm{H}}=2.5 \mathrm{~V}$, and $\mathrm{NM}_{\mathrm{L}}=1.9 \mathrm{~V}$. (f) VTCs before and after exposure to air for 5 months $\left(V_{\mathrm{DD}}=10 \mathrm{~V}\right)$

Flexibility of the hybrid inverters. The PI substrate was delaminated from the glass support using a laser lift-off (LLO) method to evaluate the flexibility of the as-fabricated inverters. Figure 4(a) shows a photograph of the ICs on a free-standing PI film and Figure 4(b) 
compares the VTCs obtained before and after delamination at $V_{\mathrm{DD}}=4,6,8$, and $10 \mathrm{~V}$. The overlap between the VTCs at each $V_{\mathrm{DD}}$ indicated that the LLO process did not adversely affect the electrical performance of the inverter.

(a)



(b)



Figure 4 Properties of the hybrid inverter before and after delamination from glass supports.

(a) Photograph of the hybrid ICs on a free-standing PI film. (b) VTCs of the hybrid complementary inverter with p- and n-channel $W / L$ values of $200 \mu \mathrm{m} / 9 \mu \mathrm{m}$ and $200 \mu \mathrm{m} / 13$ $\mu \mathrm{m}$, respectively, before and after delamination

The bending stress test was carried out by measuring VTCs by laminating the PI film devices were onto the cylindrical surfaces (Figure 5(a),(b)). With various bent radii (17.5, 12.0, and $6.0 \mathrm{~mm}$ ), no significant changes were observed. As shown in Figure 5(c)-(f), both the p- and n-channel TFTs exhibited decent transfer characteristics under the action of a bending stress. The slight $V_{\text {on }}$ shift is probably due to system error during measurement ${ }^{36,44}$. The bending stress applied on semiconductors depends not only on the bending radii but also device structure and substrate thickness. The corresponding surface strain $(\varepsilon)$ can be calculated using the following equation ${ }^{45}$

$\varepsilon=\frac{\mathrm{h}_{\mathrm{s}}}{2 R} \times 100 \%$

where $R$ is the bending radius and $h_{\mathrm{s}}$ is substrate thickness. In principle, when a substrate is bent, there is always a layer with zero bending stress and while the inner surface suffers 
compression stress, the outer surface suffers tensile stress. In this study, compared with the thickness of PI substrates $(\sim 10 \mu \mathrm{m})$, the total thickness of the other layers $(<500 \mathrm{~nm})$ was much lesser; hence, the TFTs experienced a tensile force perpendicular to the channels. In other words, the tensile force was applied in the $c$-axis direction, i.e., the preferred carriertransport direction in the herringbone packing structure of the $\mathrm{C}_{9}$-DNBDT-NW single crystal, which might decrease carrier mobility ${ }^{45}$. Meanwhile, amorphous IZO was isotopically stressed. Using Equation (2), the maximum tensile stress was estimated to be $0.08 \%$ with a bending radius of $6 \mathrm{~mm}$, at which the decrease in mobility was small $(1 \%)^{45}$; this observation is consistent with our results. In addition, the small tensile stress should have negligible effects on the amorphous IZO layer. Therefore, the identical electrical performance of the inverter under bending conditions suggests that this hybrid technology can enrich flexible electronic applications. 
(a)



(c)



(e)





(d)


Figure 5 Electrical performance of a hybrid complementary inverter under bending stress. (a)

Schematic illustration of a device under bending stress. (b) VTCs of the inverter when flat and bent to tensile radii of $17.5,12.0$, and $6 \mathrm{~mm}$. The inset shows the measurement setup with a bending radius of $6 \mathrm{~mm}$. Transfer curves of TFTs based on (c and d) $\mathrm{C}_{9}$-DNBDT-NW (W/L= $100 \mu \mathrm{m} / 19 \mu \mathrm{m})$ and (e and f) $\operatorname{IZO}(W / L=50 \mu \mathrm{m} / 24 \mu \mathrm{m})$ in the linear and saturation regions under different bending stresses.

Performance of hybrid ring oscillators. Unlike direct calculation by propagation delay, ring oscillators provide a simple and effective way to evaluate the maximum switching speed of 
larger logic gates. In a ring oscillator, each inverter delays the input signal for a specific time, which is defined as the stage propagation delay $\left(t_{\mathrm{p}}\right)$. To simplify, we assume that all inverters have the same property and the same delay time. The delay time at the output can hence be written as

$T=2 n t_{\mathrm{p}}$

where $n$ is the stage number and $T$ is the period of the ring oscillator. By measuring the operation frequency of the ring oscillator $\left(f_{\mathrm{ROSC}}\right), t_{\mathrm{p}}$ can be calculated as shown in Equation (4).

$f_{\mathrm{ROSC}}=\frac{1}{T}=\frac{1}{2 n t_{\mathrm{p}}}$

Five-stage ring oscillators were fabricated by connecting five inverters in a loop to study the propagation delay of the hybrid inverter and the availability of more complex circuits. An optical micrograph of the as-fabricated ring oscillator is shown in Figure 6(a). In each stage, the dimensions were $W / L=200 \mu \mathrm{m} / 4 \mu \mathrm{m}$ and $\Delta L=3 \mu \mathrm{m}$ for the p-channel TFT and $W / L=$ $200 \mu \mathrm{m} / 8 \mu \mathrm{m}$ and $\Delta L=1.5 \mu \mathrm{m}$ for the n-channel TFT. The electrical performance of the $\mathrm{p}$ and n-channel TFTs recorded under these conditions is shown in Figure S8. The p-channel TFT exhibited $\mu_{\text {lin }}$ and $\mu_{\text {sat }}, I_{\text {off }}$, and $I_{\text {on }} / I_{\text {off }}$ ratio of 4.4 and $1.1 \mathrm{~cm}^{2} \mathrm{~V}^{-1} \mathrm{~s}^{-1}, 10^{-13} \mathrm{~A}$, and $10^{8}$, respectively, while the corresponding values for the $\mathrm{n}$-channel TFT were 1.6 and $1.9 \mathrm{~cm}^{2} \mathrm{~V}^{-1}$ $\mathrm{s}^{-1}, 10^{-13} \mathrm{~A}$, and $10^{9}$ respectively. Note that, for the $\mathrm{p}$-channel TFT, $\mu_{\mathrm{sat}}$ value was smaller than $\mu_{\text {lin }}$, which was recently observed in the short-channel, single-crystal OSC-based TFT and attributed to velocity saturation effect, while the details are still under discussion. ${ }^{46}$

The VTC of a single inverter (Figure 6(b)) suggests that even those inverters with short channel lengths exhibit a full rail-to-rail swing, symmetric transition of $V_{\mathrm{M}} \sim 5 \mathrm{~V}$ for both forward and backward voltage sweep, and a high noise margin with an $\mathrm{NM}_{\mathrm{H}}$ of $3.2 \mathrm{~V}$ and 
$\mathrm{NM}_{\mathrm{L}}$ of $2.9 \mathrm{~V}$. The output signal from the five-stage ring oscillator at a $V_{\mathrm{DD}}$ of $10 \mathrm{~V}$ is shown in Figure 6(c). In this case, $f_{\mathrm{ROSC}}$ was $77 \mathrm{kHz}$ and thus $t_{\mathrm{p}}$ was estimated to be $1.3 \mu \mathrm{s}$. We compared several complementary ring oscillators based on solution-processed OSCs or MOSs with respect to $t_{\mathrm{p}}$ (Table 1). As there are only a few studies on plastic substrates, ring oscillators fabricated on rigid substrates were also considered. Because $f_{\text {ROSC }}$ is approximately proportional to $V_{\mathrm{DD}}{ }^{47}$, different devices were compared by converting $V_{\mathrm{DD}}$ to $10 \mathrm{~V}$ and the


the present study exhibited the fastest operation speed (i.e., the smallest $t_{\mathrm{p}}$ ) among the reports. As shown in Figure S9, the complementary ring oscillators were sufficiently fabricated over the substrate. Considering $f_{\mathrm{ROSC}}$ with different TFT dimensions, $f_{\mathrm{ROSC}}$ in the current devices was principally dominated by $L$ but subsequently by $\Delta L$. Hence, the future task for increasing $f_{\text {ROSC }}$ may include an improved technology of fine patterning, where the reduction of contact resistance should get required for advanced performances.

(a)



(b)



(c)




Figure 6 Properties of a five-stage ring oscillator. (a) Optical micrograph of a hybrid ring oscillator. The magnified image shows one stage. $W / L=200 \mu \mathrm{m} / 4 \mu \mathrm{m}$ and $\Delta L=3 \mu \mathrm{m}$ for pchannel TFT and $W / L=200 \mu \mathrm{m} / 8 \mu \mathrm{m}$ and $\Delta L=1.5 \mu \mathrm{m}$ for n-channel TFT. (b) VTCs of the hybrid inverter with $V_{\mathrm{DD}}=10 \mathrm{~V}$. (c) Output signal of the ring oscillator at $V_{\mathrm{DD}}=10 \mathrm{~V}$

Table 1 Comparison of the stage-propagation delay in ring oscillators based on solutionprocessed MOSs or OSCs

\begin{tabular}{|c|c|c|c|c|c|c|c|c|c|}
\hline $\begin{array}{l}\text { p-channel } \\
\text { material }\end{array}$ & $\begin{array}{c}\text { n-channel } \\
\text { material }\end{array}$ & Substrate & $\begin{array}{l}\text { Stage } \\
\text { No.* }\end{array}$ & $\begin{array}{l}L_{\mathrm{p}} / L_{\mathrm{n}}^{\dagger} \\
(\mu \mathrm{m})\end{array}$ & $\begin{array}{l}V_{\mathrm{DD}^{*}} \\
(\mathrm{~V})\end{array}$ & $\begin{array}{l}f_{\mathrm{ROSC}^{\S}} \\
(\mathrm{kHz})\end{array}$ & $\begin{array}{l}t_{\mathrm{p}}^{* *} \\
(\mu \mathrm{s})\end{array}$ & $\begin{array}{c}t_{\mathrm{p}(10 \mathrm{~V})^{\dagger \dagger}} \\
(\mu \mathrm{s})\end{array}$ & Ref. \\
\hline $\begin{array}{c}\mathrm{C}_{9} \text {-DNBDT- } \\
\mathrm{NW}\end{array}$ & IZO & PI & 5 & $4 / 8$ & 10 & 77 & 1.3 & 1.3 & $\begin{array}{l}\text { This } \\
\text { work }\end{array}$ \\
\hline $\begin{array}{c}\mathrm{C}_{10} \text { DNBDT- } \\
\mathrm{NW}\end{array}$ & $\begin{array}{c}\text { BASF GSID } \\
104031-1\end{array}$ & Glass & 5 & $30 / 5$ & 20 & 22 & 4.5 & 9 & 26 \\
\hline IDT-BT & IZO & Glass & 3 & $5 / 5$ & 10 & 3.2 & 52 & 52 & 33 \\
\hline P3HT & $\mathrm{ZnO}$ & $\mathrm{Si}$ & 5 & $1 / 1$ & 2 & 2.2 & 45 & 9 & 48 \\
\hline $\begin{array}{c}\text { TIPS- } \\
\text { pentacene }\end{array}$ & $\begin{array}{c}\text { BASF GSID } \\
\text { 104031-1 }\end{array}$ & PET & 5 & $70 / 70$ & 100 & 0.134 & 746 & 7460 & 49 \\
\hline TIPS-PEN & $\begin{array}{l}\text { ActivInk } \\
\text { N3300 }\end{array}$ & PEN & 19 & $5 / 5$ & 10 & 1.0 & 25 & 25 & 50 \\
\hline $\begin{array}{l}\text { ActivInk } \\
\text { P2100 }\end{array}$ & $\begin{array}{c}\mathrm{P}(\mathrm{NDI} 2 \mathrm{OD}- \\
\mathrm{T} 2)\end{array}$ & PEN & 5 & $5 / 5$ & 10 & 10 & 10 & 10 & 51 \\
\hline DPPT-TT & $\begin{array}{c}\mathrm{P}(\mathrm{NDI} 2 \mathrm{OD}- \\
\mathrm{T} 2)\end{array}$ & PEN & 7 & I & 100 & 2 & 31 & 310 & 52 \\
\hline $\mathrm{P}\left(\mathrm{T}_{0} \mathrm{~T}_{0} \mathrm{TT}_{16}\right)$ & $\begin{array}{c}\mathrm{P}(\mathrm{NDI} 2 \mathrm{OD}- \\
\mathrm{T} 2)\end{array}$ & Glass & 7 & $2.5 / 2.5$ & 1.5 & 0.14 & 500 & 75 & 53 \\
\hline $\begin{array}{l}\text { DiF-TES- } \\
\text { ADT/PS }\end{array}$ & TU-3 & Parylene & 3 & I & 10 & 0.217 & 768 & 768 & 23 \\
\hline
\end{tabular}


DiF-TES-

$$
\text { TU-3/P } \alpha M S
$$

Glass

$$
5
$$

$10 / 10$

10

1.465

68.2

$68.2 \quad 54$

ADT/PS

MOP-01

TU-3/P $\alpha M S$

Glass

5

$60 / 55$

10

0.09

1065

1065

55

*Stage No.: Stage number

${ }^{\dagger} L_{\mathrm{p}} / L_{\mathrm{n}}$ : Channel lengths for $\mathrm{n}$-channel TFT and p-channel TFT

$¥ V_{\mathrm{DD}}$ : Supply voltage

$\S_{\text {ROSC: }}$ Frequency of ring oscillator

${ }^{* * *} t_{\mathrm{p}}$ : Stage propagation delay

${ }^{\dagger} t_{\mathrm{p}(10 \mathrm{~V})}$ : Stage propagation delay at $V_{\mathrm{DD}}=10 \mathrm{~V}$ or converted $V_{\mathrm{DD}}$ to $10 \mathrm{~V}$

\section{Conclusion}

We have demonstrated organic-inorganic hybrid complementary ICs on plastic substrates by using solution-processed, high-performance semiconductors: p-type $\mathrm{C}_{9}$-DNBDT-NW single crystals and n-type amorphous IZO. Owing to their high carrier mobilities and air stability, the TFTs and the complementary inverters exhibited excellent electrical characteristics and long-term stability, and the five-stage complementary ring oscillator operated at $77 \mathrm{kHz}$ with a supply voltage of $10 \mathrm{~V}$ in air, proving the likeliest approach to high-speed complementary ICs based on the solution-processed semiconductors. Although solution-processed amorphous MOSs are usually sensitive to chemical species such as water and organic solvents, the reliable integration of the OSC-based TFTs with the pre-fabricated MOS-based TFTs shown in this paper can be attributed to the following materials viewpoints: 1) An effective protection bilayer for IZO based on a PMMA underlayer, which is solution-processable at low temperature and chemically harmless for IZO, and acts as a barrier of the undesired reaction between IZO and the diradical parylene intermediate during the formation of upper layer; 2) Water-based transferring integration of printed OSC thin films with the prefabricated IZO-based TFTs with the help of hydrophobic parylene layer; 3) Damage-free 
photolithographic processes using the dry-film, photoacid-free photoresist. The scalability of current printing methods can promise further developments of the high-speed complementary ICs for IoT applications, where it is required to reduce the contact resistance and to minimise the channel dimensions and capacitive parasitism, which needs the help of materials science.

\section{Methods}

Substrate preparation. All the devices used in this study were fabricated on PI substrates. The PI substrate was prepared by spin-coating polyamic acid (Ube Industries, Ltd.) on a glass supporter $\left(5 \times 5 \mathrm{~cm}^{2}\right)$ at $2000 \mathrm{rpm}$ for $3 \mathrm{~min}$, followed by thermal curing at $110{ }^{\circ} \mathrm{C}$ for 60 $\min , 150{ }^{\circ} \mathrm{C}$ for $30 \mathrm{~min}, 200{ }^{\circ} \mathrm{C}$ for $10 \mathrm{~min}, 250{ }^{\circ} \mathrm{C}$ for $10 \mathrm{~min}$, and $430{ }^{\circ} \mathrm{C}$ for $10 \mathrm{~min}$ on a hot plate in the air. The PI film was attached to a glass support during fabrication and delaminated using an LLO technique to achieve free-standing films for flexibility evaluation.

Fabrication of n-channel TFTs based on IZO. IZO films were fabricated using a sol-gel method. Initially, In and $\mathrm{Zn}$ precursor solutions $(0.1 \mathrm{M})$ were prepared by adding $\mathrm{In}\left(\mathrm{NO}_{3}\right)_{3} \cdot \mathrm{xH}_{2} \mathrm{O}($ Aldrich $)$ and $\mathrm{Zn}\left(\mathrm{NO}_{3}\right)_{2} \cdot \mathrm{xH}_{2} \mathrm{O}$ (Aldrich) to 2-methoxyethanol, respectively, and stirring at room temperature in the air for more than $6 \mathrm{~h}$. The IZO precursor was then prepared by mixing the In and $\mathrm{Zn}$ precursors at an $\mathrm{In} / \mathrm{Zn}$ ratio of $3 / 2$ and stirring under the conditions described above.

Both n- and p-channel TFTs exhibit a bottom-gate top-contact structure, as shown in Figure S1. Gate patterns of IZO-based TFTs were formed by photolithography with a photoresist (TLOR, Tokyo Ohka Kogyo Co., Ltd.). Cr/Au/Cr (5/25/5 nm) was deposited via thermal evaporation, followed by a lift-off process. $\mathrm{An} \mathrm{AlO}_{\mathrm{x}}$ gate dielectric layer was formed by ALD. Before IZO deposition, the substrate was treated with a UV ozone cleaner (Filgen, Inc., $\mathrm{UV} 253 \mathrm{H}$ ) for $10 \mathrm{~min}$ to remove any organic residues and improve its wettability. The IZO precursor was then spin-coated on the substrate at $500 \mathrm{rpm}$ for $5 \mathrm{~s}$ and $5000 \mathrm{rpm}$ for $30 \mathrm{~s}$, 
followed by soft baking at $150{ }^{\circ} \mathrm{C}$ for 5 min and hard baking at $370{ }^{\circ} \mathrm{C}$ for $1 \mathrm{~h}$ under ambient conditions. The IZO film was patterned with a PDM (Taiyo Ink Mgf. Co., Ltd.), and etched with oxalic acid. S/D electrodes for IZO-based TFTs and gate electrodes for $\mathrm{C}_{9}$-DNBDTNW-based TFTs (Al, $45 \mathrm{~nm}$ ) were deposited by thermal evaporation and patterned by a liftoff process based on $\mathrm{PDM}^{36}$.

Fabrication of p-channel TFTs based on C9-DNBDT-NW. After the fabrication of nchannel TFTs, a PMMA/parylene bilayer was fabricated to act as a passivation layer for nchannel TFTs and it doubled as a gate dielectric for p-channel TFTs. The PMMA layer was formed by spin coating a PMMA solution $(\mathrm{Mw}=120,000,0.56 \mathrm{wt} . \%$ in butyl acetate $)$ at 500 $\mathrm{rpm}$ for $5 \mathrm{~s}$ and then $4000 \mathrm{rpm}$ for $30 \mathrm{~s}$, followed by soft baking at $150{ }^{\circ} \mathrm{C}$ for $1 \mathrm{~h}$. The parylene layer was deposited by chemical vapour deposition.

$\mathrm{C}_{9}$-DNBDT-NW was synthesised and purified in-house; initially, a $\mathrm{C}_{9}$-DNBDT-NW solution was prepared by dissolving 0.02 wt.\% $\mathrm{C}_{9}$-DNBDT-NW in 3-chlorothiophene. The $\mathrm{C}_{9}$ DNBDT-NW single-crystal layer was printed by continuous edge casting on a super hydrophilic substrate and then transferred to the top of the PMMA/parylene dielectric layer. More details on the printing and OSC transfer methods can be found in our previous reports $^{7,38,39}$.

Fine patterns of OSC and Au S/D electrodes were achieved by a two-step patterning process based on a dry film resist with a thickness of $5 \mu \mathrm{m}$ (PDM, Taiyo Ink Mfg. Co., Ltd. $)^{40,41}$. This process is illustrated schematically in Figure S10. A PMMA layer (Mw = 120,000, 5 wt.\% in butyl acetate) was formed by spin coating at $500 \mathrm{rpm}$ for $5 \mathrm{~s}$ and $1000 \mathrm{rpm}$ for $30 \mathrm{~s}$, followed by baking at $80^{\circ} \mathrm{C}$ for 10 min before being laminated with a PDM dry film. The PDM layer was patterned by photolithography and PMMA was patterned using $\mathrm{O}_{2}$ plasma with patterned PDM as a mask. After etching the OSC or S/D electrodes, PMMA and PDM were stripped together with acetonitrile. For OSC patterning, $\mathrm{Au}(30 \mathrm{~nm})$ was deposited via thermal 
evaporation on the entire surface and it acted as a protection layer as well as S/D electrodes. Subsequently, a two-step photolithography process was conducted. Au was etched using an AURUM S-50790 (Kanto Chemical Co. Inc.) instrument and the OSC layer was etched using $\mathrm{O}_{2}$ plasma. Subsequently, a solid-state laser (Delphi Laser, Inducer-6001-P, $355 \mathrm{~nm}$ ) was used to create holes in the gate dielectrics for bottom electrodes. Next, $\mathrm{Au}(60 \mathrm{~nm})$ was deposited by thermal evaporation and patterned by two-step photolithography to form S/D electrodes and conduction terminals for the bottom electrodes. Finally, the substrate was annealed at $110{ }^{\circ} \mathrm{C}$ for $1 \mathrm{~h}$ to remove the solvent used during fabrication.

Device characterisation. The electrical properties of the fabricated hybrid inverters were measured under ambient and dark conditions. The static properties of the TFTs and the VTCs of the inverters were measured using a semiconductor parameter analyser (Keithley, 4200SCS). The output signals of the ring oscillators were recorded using an oscilloscope (Tektronix, MDO3014). Micrographs of the complementary inverter and ring oscillator were acquired using an optical microscope. An FIB-SEM (JEOL, JIB-4700F) was used to observe the edge conditions of the gate electrodes in p-channel TFTs.

Carrier mobility $(\mu)$ and $V_{\text {th }}$ were determined by measuring the dependence of drain current ( $\left.I_{\mathrm{D}}\right)$ on $V_{\mathrm{G}}$ and fitting with the following equations.

In the linear region,

$I_{\mathrm{D}}=\frac{\mu_{\text {lin } W C_{\mathrm{i}}}}{L} V_{\mathrm{D}}\left(V_{\mathrm{G}}-V_{\mathrm{th}}\right)$

In the saturation region,

$I_{\mathrm{D}}=\frac{\mu_{\mathrm{sat}} W C_{\mathrm{i}}}{2 L}\left(V_{\mathrm{G}}-V_{\mathrm{th}}\right)^{2}$

$C_{\mathrm{i}}$ is determined by capacitance-voltage measurements at $10 \mathrm{kHz}$. The values of $C_{\mathrm{i}}$ for the ring oscillators used in this study were $88.5 \mathrm{nF} \mathrm{cm}^{-2}$ for $\mathrm{n}$-channel $\left(\mathrm{AlO}_{\mathrm{x}}, 80 \mathrm{~nm}\right)$ and $13.2 \mathrm{nF} \mathrm{cm} \mathrm{cm}^{-2}$ 
for p-channel (PMMA, 10 nm; parylene, 190 nm); for inverters discussed in other parts (excepted the ring oscillators, Figure 6, Figure S8, and Figure S9) $122.5 \mathrm{nF} \mathrm{cm}^{-2}$ for n-channel $\left(\mathrm{AlO}_{\mathrm{x}}, 63 \mathrm{~nm}\right)$ and $22.1 \mathrm{nF} \mathrm{cm}{ }^{-2}$ for $\mathrm{p}$-channel (PMMA, $\sim 10 \mathrm{~nm}$; parylene, $\sim 120 \mathrm{~nm}$ ).

\section{References}

1. Chang, J. S., Facchetti, A. F. \& Reuss, R. A circuits and systems perspective of organic/printed electronics: review, challenges, and contemporary and emerging design approaches. IEEE Trans. Emerg. Sel. Topics Circuits Syst. 7, 7-26 (2017).

2. Minemawari, H. et al. Inkjet printing of single-crystal films. Nature 475, 364-367 (2011).

3. Diao, Y. et al. Solution coating of large-area organic semiconductor thin films with aligned single-crystalline domains. Nat. Mater. 12, 665-671 (2013).

4. Arai, S., Inoue, S., Hamai, T., Kumai, R. \& Hasegawa, T. Semiconductive single molecular bilayers realized using geometrical frustration. Adv. Mater. 30, 1707256 (2018).

5. Peng, B., Wang, Z. \& Chan, P. K. L. A simulation-assisted solution-processing method for a large-area, high-performance $\mathrm{C}_{10}$-DNTT organic semiconductor crystal. J. Mater. Chem. C 4, 8628-8633 (2016).

6. Yamamura, A. et al. Wafer-scale, layer-controlled organic single crystals for high-speed circuit operation. Sci. Adv. 4, eaao5758 (2018).

7. Kumagai, S. et al. Scalable fabrication of organic single-crystalline wafers for reproducible TFT arrays. Sci. Rep. 9, 15897 (2019).

8. Duan, S. et al. Solution-Processed Centimeter-Scale Highly Aligned Organic Crystalline Arrays for High-Performance Organic Field-Effect Transistors. Adv. Mater. 32, 1908388 (2020). 
9. Okamoto, T. et al. Robust, high-performance n-type organic semiconductors. Sci. Adv. 6, eaaz0632 (2020).

10. Thomas, S. R., Pattanasattayavong, P. \& Anthopoulos, T. D. Solution-processable metal oxide semiconductors for thin-film transistor applications. Chem. Soc. Rev. 42, 6910-6923 (2013).

11. Fortunato, E., Barquinha, P. \& Martins, R. Oxide semiconductor thin-film transistors: a review of recent advances. Adv. Mater. 24, 2945-2986 (2012).

12. Banger, K. K. et al. Low-temperature, high-performance solution-processed metal oxide thin-film transistors formed by a 'sol-gel on chip' process. Nat. Mater. 10, 45-50 (2011).

13. Kim, M. G., Kanatzidis, M. G., Facchetti, A. \& Marks, T. J. Low-temperature fabrication of high-performance metal oxide thin-film electronics via combustion processing. Nat. Mater. 10, 382-388 (2011).

14. Kim, Y.-H. et al. Flexible metal-oxide devices made by room-temperature photochemical activation of sol-gel films. Nature 489, 128-132 (2012).

15. Ong, B. S., Li, C., Li, Y., Wu, Y. \& Loutfy, R. Stable, solution-processed, high-mobility ZnO thin-film transistors. J. Am. Chem. Soc. 129, 2750-2751 (2007).

16. Hosono, H. Ionic amorphous oxide semiconductors: material design, carrier transport, and device application. J. Non-Cryst. Solids 352, 851-858 (2006).

17. Shiwaku, R. et al. Printed 2 V-operating organic inverter arrays employing a smallmolecule/polymer blend. Sci. Rep. 6, 1-7 (2016).

18. Shiwaku, R. et al. Printed organic inverter circuits with ultralow operating voltages. Adv. Electron. Mater. 3, 1600557 (2017).

19. Watson, C. P., Brown, B. A., Carter, J., Morgan, J. \& Taylor, D. M. Organic ring oscillators with sub-200 ns stage delay based on a solution-processed p-type semiconductor blend. Adv. Electron. Mater. 2, 1500322 (2016). 
20. Kim, K. H., Kim, Y.-H., Kim, H. J., Han, J.-I. \& Park, S. K. Fast and stable solutionprocessed transparent oxide thin-film transistor circuits. IEEE Electron Device Lett. 32, $524-526(2011)$.

21. He, G., Li, W., Sun, Z., Zhang, M. \& Chen, X. Potential solution-induced HfAlO dielectrics and their applications in low-voltage-operating transistors and high-gain inverters. RSC Adv. 8, 36584-36595 (2018).

22. Kim, S. H., Lee, S. H., Kim, Y. G. \& Jang, J. Ink-jet-printed organic thin-film transistors for low-voltage-driven CMOS circuits with solution-processed $\mathrm{AlO}_{\mathrm{x}}$ gate insulator. IEEE Electron Device Lett. 34, 307-309 (2013).

23. Takeda, Y. et al. Fabrication of ultra-thin printed organic TFT CMOS logic circuits optimized for low-voltage wearable sensor applications. Sci. Rep. 6, 25714 (2016).

24. Sawada, T. et al. Low-voltage complementary inverters using solution-processed, highmobility organic single-crystal transistors fabricated by polymer-blend printing. Appl. Phys. Lett. 117, 033301 (2020).

25. Yamamura, A. et al. Painting integrated complementary logic circuits for single-crystal organic transistors: a demonstration of a digital wireless communication sensing tag. $A d v$. Electron. Mater. 3, 1600456 (2017).

26. Uno, M. et al. Short-channel solution-processed organic semiconductor transistors and their application in high-speed organic complementary circuits and organic rectifiers. $A d v$. Electron. Mater. 1, 1500178 (2015).

27. Chen, C., Yang, Q., Chen, G., Chen, H. \& Guo, T. Solution-processed oxide complementary inverter via laser annealing and inkjet printing. IEEE Trans. Electron Devices 66, 4888-4893 (2019). 
28. Rockelé, M. et al. Low-temperature and scalable complementary thin-film technology based on solution-processed metal oxide n-TFTs and pentacene p-TFTs. Org. Electron. 12, 1909-1913 (2011).

29. Ji, S.-B., Chung, S., Kwon, S.-K. \& Hong, Y. Solution-processed organic/inorganic hybrid CMOS-type inverter. SID Symp. Dig. Tech. Pap. 42, 1563-1566 (2011).

30. Isakov, I. et al. Hybrid complementary circuits based on p-channel organic and n-channel metal oxide transistors with balanced carrier mobilities of up to $10 \mathrm{~cm}^{2} / \mathrm{Vs}$. Appl. Phys. Lett. 109, 263301 (2016).

31. Hong, Y. et al. Solution-process oxide TFTs for hybrid low-voltage CMOS applications. ECS Trans. 67, 51-58 (2015).

32. Kumagai, S. et al. Solution-processed organic-inorganic hybrid CMOS inverter exhibiting a high gain reaching 890. Org. Electron. 48, 127-131 (2017).

33. Pecunia, V., Banger, K., Sou, A. \& Sirringhaus, H. Solution-based self-aligned hybrid organic/metal-oxide complementary logic with megahertz operation. Org. Electron. 21, $177-183$ (2015).

34. Pecunia, V. et al. Trap healing for high-performance low-voltage polymer transistors and solution-based analog amplifiers on foil. Adv. Mater. 29, 1606938 (2017).

35. Mitsui, C. et al. High-performance solution-processable N-shaped organic semiconducting materials with stabilized crystal phase. Adv. Mater. 26, 4546-4551 (2014).

36. Wei, X. et al. Solution-processed flexible metal-oxide thin-film transistors operating beyond 20 MHz. Flex. Print. Electron. 5, 015003 (2020).

37. Wei, X., Kumagai, S., Sasaki, M., Watanabe, S. \& Takeya, J. Stabilizing solutionprocessed metal oxide thin-film transistors via trilayer organic-inorganic hybrid passivation. AIP Adv. 11, 035027 (2021). 
38. Soeda, J. et al. Inch-size solution-processed single-crystalline films of high-mobility organic semiconductors. Appl. Phys. Express 6, 076503 (2013).

39. Makita, T. et al. High-performance, semiconducting membrane composed of ultrathin, single-crystal organic semiconductors. Proc. Natl. Acad. Sci. U.S.A. 117, 80-85 (2020).

40. Wei, X. \& Shibasaki, Y. A novel photosensitive dry-film dielectric material for high density package substrate, interposer and wafer level package. in 2016 IEEE 66th Electronic Components and Technology Conference (ECTC) 159-164 (2016).

41. Okamoto, D., Shibasaki, Y., Shibata, D. \& Hanada, T. New photosensitive dielectric material for high-density RDL with ultra-small photo-vias and high reliability. in 2018 51st International Symposium on Microelectronics (IMAPS) 2018, 000466-000469 (2018).

42. Kim, B. et al. High-speed, inkjet-printed carbon nanotube/zinc tin oxide hybrid complementary ring oscillators. Nano Lett. 14, 3683-3687 (2014).

43. Uemura, T. et al. On the extraction of charge carrier mobility in high-mobility organic transistors. Adv. Mater. 28, 151-155 (2016).

44. Watanabe, S. et al. Surface doping of organic single-crystal semiconductors to produce strain-sensitive conductive nanosheets. Adv. Sci. 8, 2002065 (2021).

45. Kubo, T. et al. Suppressing molecular vibrations in organic semiconductors by inducing strain. Nat. Commun. 7, 11156 (2016).

46. Peng, B. et al. Crystallized monolayer semiconductor for ohmic contact resistance, high intrinsic gain, and high current density. Adv. Mater. 32, 2002281 (2020).

47. Biabanifard, S., Largani, S. M. H. \& Asadi, S. Delay time analysis of combined CMOS ring oscillator. ELELIJ 4, 53-64 (2015).

48. Hong, K. et al. Aerosol jet printed, sub-2 V complementary circuits constructed from pand n-type electrolyte gated transistors. Adv. Mater. 26, 7032-7037 (2014). 
49. Kempa, H. et al. Complementary ring oscillator exclusively prepared by means of gravure and flexographic printing. IEEE Trans. Electron Devices 58, 2765-2769 (2011).

50. Smaal, W. et al. Complementary integrated circuits on plastic foil using inkjet printed nand p-type organic semiconductors: fabrication, characterization, and circuit analysis. Org. Electron. 13, 1686-1692 (2012).

51. Baeg, K.-J. et al. Low-voltage, high speed inkjet-printed flexible complementary polymer electronic circuits. Org. Electron. 14, 1407-1418 (2013).

52. Mandal, S. et al. Fully-printed, all-polymer, bendable and highly transparent complementary logic circuits. Org. Electron. 20, 132-141 (2015).

53. Herlogsson, L., Crispin, X., Tierney, S. \& Berggren, M. Polyelectrolyte-gated organic complementary circuits operating at low power and voltage. Adv. Mater. 23, 4684-4689 (2011).

54. Takeda, Y. et al. High-speed complementary integrated circuit with a stacked structure using fine electrodes formed by reverse offset printing. ACS Appl. Electron. Mater. 2, 763-768 (2020).

55. Takeda, Y. et al. Printed organic complementary inverter with single SAM process using a p-type D-A Polymer semiconductor. Appl. Sci. 8, 1331 (2018).

\section{Acknowledgement}

We would like to thank Editage (www.editage.com) for English language editing.

\section{Author contributions}

J.T. and S.W. conceived the study. X.W. designed the integration process and carried out device fabrication, characterisation studies, and the related data analysis. S. K. contributed to the process design and data analysis of metal-oxide semiconductors and the passivation process. T.M. contributed to the fabrication and patterning of organic semiconductors. K.T. 
discussed the IZO preparation process. A.Y. contributed to the design of complementary circuits. All authors analysed and interpreted the data and wrote the manuscript.

\section{Competing interests}

The authors declare no competing interests. 


\section{Supplementary Files}

This is a list of supplementary files associated with this preprint. Click to download.

- CMOSweiSI210907FV.pdf 